\title{
Tilting and Beam-shaping for Traffic Load Balancing in WCDMA Network
}

\author{
Jiayi Wu, John Bigham, Peng Jiang, John Peter Neophytou \\ Department of Electronic Engineering, Queen Mary University of London \\ Mile End Road, London E1 4NS UK
}

\begin{abstract}
This paper ${ }^{1}$ summarizes recent developments in semi-smart antenna systems for geographic load balancing in cellular mobile communication systems by cooperatively changing the antenna radiation patterns and compares the performance with cooperatively down tilted antennas. Cooperative coverage is advantageous, e.g. in the presence of hotspots. For semi-smart antennas the ideal shape for a cell in the context of its neighbours is determined using the Bubble Oscillation Algorithm (BOA) and the pattern is then given to the semi-smart antenna for further radiation pattern synthesis. The adjustment process differs for different antenna systems and mostly depends on the antenna's physical capabilities on pattern shaping.
\end{abstract}

This paper then considers the special case of adaptively tilting through the use of Electrical Down-Tilt (EDT) antennas, and compares the system capacity performance between EDT and semi-smart antennas for traffic load balancing. Coverage under load balancing is optimal with respect to the whole network, rather than just for individual antennas. The results show that both the adaptive approaches give benefit, with different site costs in relation to practical antenna system complexity.

Index Terms - semi-smart antenna, Electrical Down-Tilt (EDT), Wideband CDMA (WCDMA), Frequency Division Duplexing (FDD).

\section{INTRODUCTION}

Non-uniform distribution of mobile handsets can present problems for resource allocation at base stations (BS). The moving traffic can form hotspots (e.g. at a stadium, road traffic jam) and if the operator's network has not much extra resource for additional subscribers, denial of service or decrease of connection throughput could cause dissatisfaction. Many techniques have been developed to make use of limited radio frequency resource more effectively. In the MAC layer work focuses on dynamic channel reallocation, and in physical layer cell splitting is a traditional method. However, at the physical layer dynamic cell shaping using semi-smart and adaptive antennas was proposed in [1]. Using semi-smart antennas is a way to give extra flexibility to network

\footnotetext{
${ }^{1}$ This work was supported through the US Office of Naval Research grant BAA 03-001 Provision of Quality of Service on Wireless Networks, grant reference N00014-03-1-0323 and through the Ofcom Contract No. 830000081
}

operators as the signal energy distribution is now more manageable and can more readily accommodate the traffic demand by fitting the radiation patterns in real time to the optimal shape for maximising network capacity, or maximising some other criterion. In the original work the optimisation was achieved by notional negotiation between Base Stations (BS) [1]. The algorithm has some timing problems when applied to fully adaptive antennas so later the bubble oscillation algorithm (BOA) is designed. The BOA is robust and appears to be effective on computing optimal shapes quickly. For more details on BOA please refer to [3].

A semi-smart antenna system with multiple beam-formers, under control of specially designed antenna pattern synthesis algorithm, can be used to generate radiation patterns approximating to that determined by the BOA. Evaluations under system level WCDMA simulation can be found in [2]. The semi-smart antenna system can also be used together with EDT, which makes the system more efficient when continuously applying synthesized power pattern.

Antenna down-tilt, as a comparatively cost-efficient way to optimize network coverage and performance, has received wide attentions recently. By changing the outer frontier of a sector through tilting up or down the antenna radiation angle, a network operator has the capability to optimize power and interference distribution, which is an essential optimization technique in CDMA-based network. Down-tilt can be done via either Mechanical Down-Tilt (MDT) or Electrical DownTilt (EDT). EDT is generally more efficient as the elevation pattern can be controlled almost constant during antenna tilt, whereas MDT causes more notable changes to radiation pattern [4][6] and affects performance. For this reason, the paper focuses on Electrical Down-Tilt.

For existing deployed systems EDT is arguably more commercially viable than beam-forming antenna systems as the upgrade in hardware is more cost-efficient. The argument does not apply to systems that are not yet deployed such as WiMax. Studies on tilting in WCDMA have been carried out and the effects of tilt angle to various issues have been generalized [6]. Some empirical models have been given suggesting the optimal angle to tilt, depending on site spacing, antenna height, and a reference traffic distribution profile. However, the temporal fluctuations in traffic and the changes in locations of traffic hotspots due to unexpected events still raises challenge to the adaptability of a network. Adaptive tilting system could be a solution to, e.g. [5], and the literature reports enhanced capacity. This paper is a step in the direction of evaluating the benefits of tilting adaptively to 
accommodate real-time geographically unbalanced traffic and comparing it with the semi-smart approach, specifically in WCDMA FDD. The capacity improvement results from the decreased level of interference, as in a CDMA network the reduced interference will be converted to extra system capacity. The interference is reduced because the main-beam of the antenna is shaped to direct energy towards the desired MS (in coordination with a back off if required from neighbouring base stations) A MS outside the lobe of the beam will not receive signal from the sector antenna, thus will receive less interference from the downlink and cause less on the uplink. The system is designed to ensure that there are no holes in the coverage.

\section{ADAPTIVE TILTING APPROACH}

Adaptive tilting in WCDMA is challenging, as basically any radio energy in a $5 \mathrm{MHz}$ FDD band (either downlink or uplink) is regarded by the system as interference. The duplicate uses of same frequency by adjacent cells, makes the capacity model complicated for a straightforward solution to be worked out. Traditional techniques on tilt angle selection are based on empirical study of performance of different tilt angle, concerning coverage, throughput and service probability [6]. In [5] an adaptive tilt scheme is suggested. The model aims to find optimal tilt angle when load factors of two adjacent sectors are just balanced through a tilt-down and tilt-up search process. Capacity enhancement is reported for different hotspot positions. Although there is no direct indication that a tilt angle is optimal when two sectors are balanced, analysis through simulation shows that given a fixed traffic distribution, the service probability curve of a sector (percentage of served subscriber) is almost cap-shaped when the tilt angle increases from 0 . This suggests that a search process could be an effective method to help adaptive tilting when a mathematical solution is not available, as there are unlikely to be multiple extremas. However, the search process needs to be carefully managed, as the minimum load factor difference between neighbouring sectors is not the goal of the search. The method should be in fact capacity driven.

The study proposes a downlink-based search method for adaptive tilting. There are several reasons for the focus on downlink. Firstly, in an urban environment the downlink throughput is statistically lower than that of uplink due to decreased orthogonal code efficiency caused by multipath propagation. Secondly, the capacity of a WCDMA network is restricted by sector antenna transmission power, which means generally downlink rules the highest possible capacity. So the uplink is arguably not obviously affecting the capacity-driven search. Thirdly, the speed of search method is vital in practical application for real-time adaptive down-tilt. The chosen method is designed to reach maximum downlink capacity through tilt angle adjustment. The adjustment of tilt angle aims to balance the downlink power-based load factor, and is regulated by a capacity enhancement criterion.
The downlink load factor can be expressed in either total transmission power or sum of connection load:

$$
\begin{aligned}
& \eta_{D L}=\frac{P_{\text {tran }}}{P_{\text {Max }}} \\
& \eta_{D L}=\sum_{j=1}^{N} v_{j} \cdot \frac{\left(E_{b} / N_{0}\right)_{j}}{W / R_{j}} \cdot\left[\left(1-\alpha_{j}\right)+i_{j}\right]
\end{aligned}
$$

$N$ is the number of mobiles, $E_{b} / N_{0}$ is signal to noise ratio, $v_{j}$ is voice activity factor, $W / R_{j}$ is processing gain, $\alpha$ is orthogonality factor. In the second load factor form $i_{j}$, is the other cell to own cell interference ratio which eats up the load headroom when increases. Tilting down antenna can reduce $i_{j}$, as the interference from neighbour decreases because the upper lobe does not radiate as much power as the central lobe, thus capacity enhancement is possible. Conversely an overtilt will decrease the coverage and lead to the decrease of capacity, as the number of mobiles within range is limited.

The search method is then designed as this: in a hexagonal cellular network, a sector tilts cooperatively with its opposite neighbouring sector, aiming to maximize service probability in the two sectors when traffic distribution is unbalanced. Here only interference from the opposite neighbouring sector is considered, as interference from that direction is most prominent. The two sectors are firstly at tilt angle 0 which means the main beam is in parallel to earth plane. The transmission power of the two sectors marks the load of each sector. The service probability of mobiles within the two sectors' coverage is used as a tilt indicator. If the service probability is not above a set threshold (e.g. 90\%), the sector with the bigger power load factor will try tilting down a step (e.g.0.1 degree), and the transmission power and service probability revaluated. The down-tilt process goes on as long as service probability improves, and a maximum tilt amount should be defined to prevent coverage holes based on site spacing setting and antenna height. The tilt search process goes to another sector when load factor of that sector is bigger, and the process stops when tilting down either will not improve, or a service probability target has been met, or both sector has reached the maximum tilt angle. There is no obvious need of tilting-up during the process, because the angle starts from 0 and tilting-down is likely only going to improve the service probability before extreme is found. Side-lobe of antenna can pose a problem when an antenna's tilted down too much, but since the search process starts from a 0 angle, the impact is ignorable.

Simulation is done using the search method described above. The result is given in section IV.

\section{COVERAGE SHAPING}

Semi-smart antenna, especially those with multiple beamformers, can be used to provide traffic load balancing in a distinctive manner that is essentially not in direct relation to radio air interface characteristics. Given the flexibility to generate complicated patterns, the ideal cell contours that 
distribute demand equally among cells are able to be approximated. BOA has been proved to be effective in finding those ideal shapes [3], without causing coverage loss. A dedicated antenna synthesis algorithm is also designed [8] to provide fast pattern re-synthesis ability.

Apart from the primary geographic load balancing benefits, beam shaping can also provide flexible antenna directivity. The main-beam of the antenna can be shaped to focus on desired demand area to provide higher antenna gains. The interference to other sector can also be minimised through minimising transmission antenna gain on undesired directions. This 360 degree direction-selective energy control capability makes coverage shaping very advantageous in CDMA-based network.

Both adaptive tilting and real-time shaping are traffic load balancing techniques. The use of traffic load balancing in WCDMA enables the system to adapt to time-varying unbalanced traffic. There is still difference between the two techniques. While adaptive tilting aims to restore the load headroom consumed by interference to increase capacity, coverage shaping enables geographically distributing traffic load among cells equally and also provides interference reduction favourably.

System level simulation are done to compare the performance of different adaptive systems, the results are shown in the next section.

\section{SIMULATION RESULTS}

Two simulations are described, one for a WCDMA FDD network applying adaptive tilting and another for a network where semi-smart antennas are used. Both results are compared with the performance of a conventional network, where adaptive optimization is not applied to maximise system capacity and tilt angle is fixed. The major uplink and downlink configuration parameters are given in table. 1 .

Table.1 Simulation Parameters

\begin{tabular}{lll}
\hline Site spacing & $d$ & $1.7 \mathrm{~km}$ \\
Cell radius & $r$ & $1 \mathrm{~km}$ \\
Antenna altitude & $h_{b}$ & $30 \mathrm{~m}$ \\
Mobile altitude & $h_{m}$ & $1.5 \mathrm{~m}$ \\
WCDMA chip rate & $W$ & $3840 \mathrm{kbps}$ \\
Bit rate & $R$ & $12.20 \mathrm{kbps}$ \\
DL SNR (ITU Pedestrian A) & $E_{b} / N_{0}$ & $12.6 \mathrm{~dB}$ \\
UL SNR (ITU Pedestrian A) & $E_{b} / N_{O}$ & $8.9 \mathrm{~dB}$ \\
Voice Activity & $v$ & 1 \\
Max sector transmission power & $P_{M A X-D L}$ & $43 \mathrm{dBm}$ \\
Max UE transmission power & $P_{M A X-U L}$ & $21 \mathrm{dBm}$ \\
Max downlink load & $\eta_{D L}$ & 0.8 \\
Max uplink load & $\eta_{U L}$ & 0.6 \\
Pilot threshold & $P_{M I N}$ & $-80 \mathrm{dBm}$ \\
Soft-handover threshold & $P_{S H O}$ & $-74 \mathrm{dBm}$
\end{tabular}

A scenario is designed as User Equipment (UE) movement forms hotspots traffic. 100 snapshots of the network are taken during simulation. Each snapshot represents the positions after an interval of 60 seconds. (Smaller time intervals have been used in other simulations and the results are similar.) Initially the MS are uniformly distributed and move randomly and the $\mathrm{BS}$ is at the centre of the hexagons in the assumed hexagonal tessellation. To emulate the forming of unbalanced traffic, some of the mobiles gradually coalesce into hot-spots. More precisely, the network configuration is:

- 100 Node-B within the network each has 6 sector antennas.

- There are totally 50,000 UE within the network and most of UEs are always moving.

10 hotspots form during the simulation and each has a population of 2,000 subscribers so $40 \%$ of the subscribers are within hotspots at the end. The relative location of each MS within a hotspot follows a normal distribution with a standard deviation of half the cell radius.

- A negative exponential call model is used for all the MS and the average call time is 120 seconds and call inter-arrival time is 720 seconds.

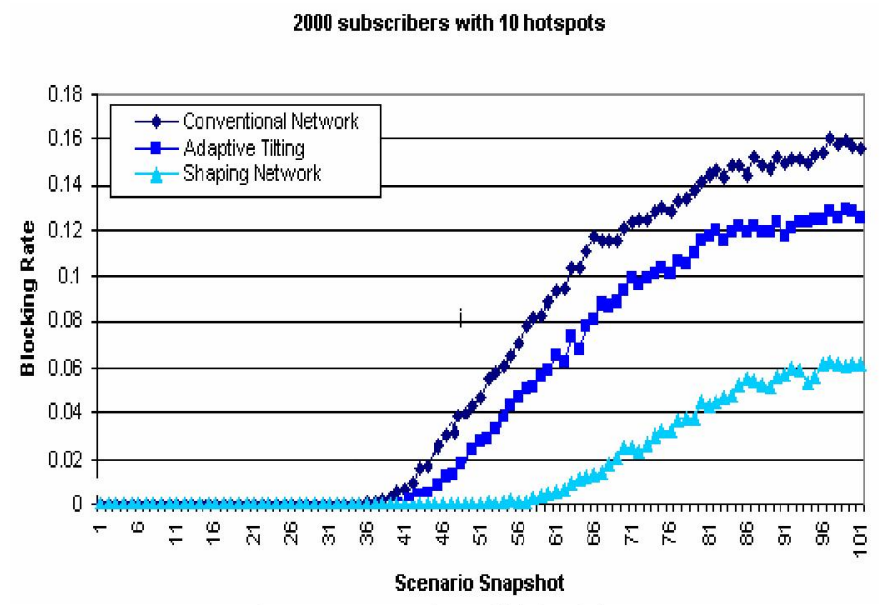

Fig 1. Network call blocking rate

Fig. 1 compares the call block rate between three networks: the conventional network with a fixed tilt angle of 1.5 degree; the network where traffic is geographically balanced using semi-smart antennas and also applies the same fixed tilt angle; and the network where adaptive EDT is applied. The results show the semi-smart system has the best performance throughout 100 snapshots. Adaptive tilting also gives performance enhancement, but does not perform better.

The results given in this paper try to show the benefit of those adaptive approaches on time-varying unbalanced traffic distribution and compare the general performance difference, rather than quantifying the enhancement percentage. The improvement rate strongly depends on scenario specification such as hotspots location, traffic distribution and network scale, site configuration, antenna main lobe half-power width, etc. Relations between these factors can be found in details in [6].

Network coverage snapshots are given here to help illustrate how the cooperative coverage shaping technique 
works. In Fig. 2 each cell is shaped. The contours are where maximum acceptable path loss is reached and can generally represent the azimuth radiation pattern of a sector. The tilt angle of antenna is fixed during simulation and elevation pattern nearly constant. The traffic load is actually balanced in topology level where a near optimal division of demands is achieved. Splines are fitted to the ideal shape computed by the BOA and then an antenna pattern synthesis program is applied to best fit the ideal pattern. Performance results are based on the synthesized pattern of a semi-smart antenna with multiple beam-formers.

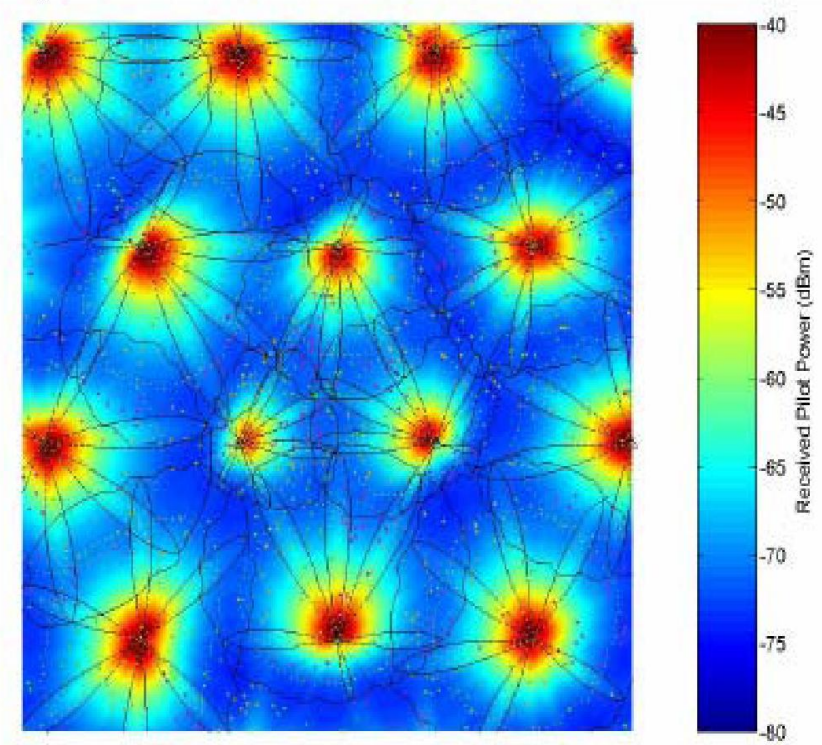

Fig.2 Real-time shaping in a WCDMA network

Adaptive tilting will also change the coverage of a sector. But a maximum tilt angle is set to avoid coverage hole.

\section{CONCLUSION}

Two techniques on traffic load balancing have been compared in this paper. Capacity enhancement is found in each case.

Coverage shaping based on a semi-smart antenna provides the best performance enhancement, as the BOA finds optimal boundaries (and soft handover boundaries) to accommodate the heterogeneous demand. This concept is applicable to most wireless technologies and with many variants of antenna system it has a great potential, especially for new deployments. Recent work has also shown significant advantages when the BS in a network is allowed to move [5]. The physical specification of antenna affects the way BOA works and the improvement is also constrained by the physical characteristics of the antenna system used.

Adaptive tilting system shows a more limited but still useful enhancement of system level capacity that can be achieved comparatively cheaply. However, the performance enhancement of adaptively tilting is determined by the air interface characteristics of a network. For example, tilt angle is an important parameter in GSM network site planning and affects the frequency reuse distance, and adaptive tilting may destroy the planned scheme. In WCDMA, adaptive tilting fits well with the technology as it gives interference reduction and can directly lead to capacity gains.

\section{ACKNOWLEDGEMENT}

We would like to thank the US Office of Naval Research grant BAA 03-001 Provision of Quality of Service on Wireless Networks, grant reference N00014-03-1-0323 and Ofcom, Proposal Reference No: C31400/004, for their support.

\section{REFERENCES}

[1] L. Du, J. Bigham, L. Cuthbert, Towards intelligent geographic load balancing for mobile cellular networks Systems, Man and Cybernetics, Part C, IEEE Transactions on, Volume: 33 , Issue: 4 , Nov. 2003, Pages: $480-491$

[2] L. Du, J. Bigham, L. Cuthbert, 2005 "Geographic Load Balancing for WCDMA Mobile Networks Using a Bubble Oscillation Algorithm", WCNC 2005, New Orleans

[3] L. Du, J. Bigham, and L. Cuthbert, "A bubble oscillation algorithm for distributed geographic load balancing in mobile networks," in The Twenty-third Annual Joint Conference of the IEEE Computer and Communications, IEEE INFOCOM'2004. Hong Kong: IEEE, March 2004.

[4] Niemela, J.; Isotalo, T.; Borkowski, J.; Lempiainen, J.; "Sensitivity of optimum downtilt angle for geographical traffic load distribution in WCDMA" Vehicular Technology Conference, 2005. VTC-2005-Fall. 2005 IEEE 62nd. Volume 2, 25-28 Sept., 2005 Page(s):1202 - 1206

[5] Pettersen, M.; Braten, L.E.; Spilling, A.G.; "Automatic antenna tilt control for capacity enhancement in UMTS FDD" Vehicular Technology Conference, 2004, VTC2004-Fall. 2004 IEEE $60^{\text {th. }}$ Volume 1, 26-29 Sept. 2004 Page(s):280 - 284 Vol. 1

[6] Niemela, J.; Isotalo, T.; Lempiainen, J.; "Optimum Antenna Downtilt Angles for Macrocellular WCDMA Network" EURASIP Journal on Wireless Communications and Networking 2005:5, 816-827

[7] John Bigham, Jiayi Wu, "Real time shaping of wireless coverage patterns when both terminal and base units move", Military Communications Conference, 2005. MILCOM 2005. IEEE, 17-20 Oct. 2005 Page(s): 1 - 7

[8] Nahi, P.; Parini, C.; Lin Du; Bigham, J.; Cuthbert, L.; "Cell shaping using pattern synthesis for a distributed load balancing scheme in cellular networks" Wireless Communications and Networking, 2003. WCNC 2003. 2003 IEEE Volume 1, 16-20 March 2003 Page(s):93 - 97 vol.1 